Weili Li* and Bo Yang

\title{
Crystal structure of $2,2^{\prime}$-[ethane- 1,2-diylbis(azanylylidenemethylylidene)] bis(6-chlorophenol), $\mathrm{C}_{16} \mathrm{H}_{14} \mathrm{Cl}_{2} \mathrm{~N}_{2} \mathrm{O}_{2}$
}

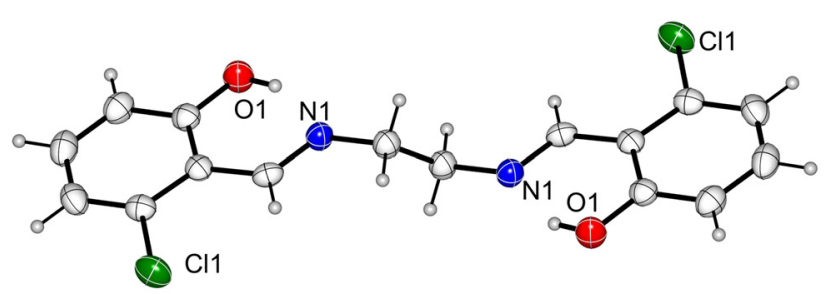

https://doi.org/10.1515/ncrs-2020-0642 Received December 27, 2020; accepted xxx; published online February 11, 2021

\begin{abstract} $\mathrm{C}_{16} \mathrm{H}_{14} \mathrm{Cl}_{2} \mathrm{~N}_{2} \mathrm{O}_{2}$, monoclinic, $P 2_{1} / n$ (no. 14), $a=6.8492(14) \AA$, $b=11.515(2) \AA, c=10.018(2) \AA, \beta=107.11(3)^{\circ}, V=755.2(3) \AA^{3}$, $Z=2, R_{g t}(F)=0.0592, w R_{r e f}\left(F^{2}\right)=0.1369, T=293(2) \mathrm{K}$.
\end{abstract}

CCDC no.: 2052614

The molecular structure is shown in the Figure. Table 1 contains crystallographic data and Table 2 contains the list of the atoms including atomic coordinates and displacement parameters.

\section{Source of material}

2-Chloro-6-hydroxybenzaldehyde and $30 \mathrm{ml}$ of anhydrous methanol were added in a conical flask, monitored with a temperature probe and warmed up to $60^{\circ} \mathrm{C}$ with stirring. Then $1.0 \mathrm{mmol}$ of ethylenediamine was dropwisely added. Over $30 \mathrm{~min}$ the mixture gradually turned to light yellow, and was refluxed for another $16 \mathrm{~h}$. The obtained solution was filtered and the filtrate was left to crystallise in an open vessel at room temperature. Colorless plates crystals were isolated from the yellow mother liquor.

*Corresponding author: Weili Li, Department of Chemical Science and Technology, Kunming University, Yunnan, Kunming 65200, P. R. China, E-mail: Iwlkmu@163.com. https://orcid.org/0000-00030700-3127

Bo Yang, Department of Chemical Science and Technology, Kunming University, Yunnan, Kunming 65200, P. R. China
Table 1: Data collection and handling.

\begin{tabular}{ll}
\hline Crystal: & Yellow block \\
Size: & $0.18 \times 0.16 \times 0.12 \mathrm{~mm}$ \\
Wavelength: & Mo $K \alpha$ radiation $(0.71073 \AA)$ \\
$\mu:$ & $0.44 \mathrm{~mm}^{-1}$ \\
Diffractometer, scan mode: & Bruker D8/APEX2, $\omega$ \\
$\theta_{\text {max }}$, completeness: & $26.4^{\circ},>99 \%$ \\
$N(h k l)_{\text {measured }}, N\left(h k l l_{\text {unique }}, R_{\text {int }}:\right.$ & $3010,1550,0.060$ \\
Criterion for $I_{\text {obs }}, N(h k l)_{\mathrm{gt}}:$ & $l_{\text {obs }}>2 \sigma\left(l_{\text {obs }}\right), 1110$ \\
$N(\text { param })_{\text {refined }}:$ & 101 \\
Programs: & Bruker [1], Olex2 [2], SHELX [3, 4] \\
\hline
\end{tabular}

\section{Experimental details}

The structure was solved with the Olex2 program [2] as an interface with the SHELXT and SHELXL programs [3, 4]. All $\mathrm{H}$ atoms were placed in geometrically idealized positions and refined using a riding model, with $\mathrm{O}-\mathrm{H}=0.84$ (phenolic hydroxyl), $0.95 \AA$ (benzene), and with $U_{\text {iso }}(\mathrm{H})=1.2 U_{\text {eq }}(\mathrm{C})$ for $\mathrm{H}$ atoms on phenolic hydroxyl and benzene.

Table 2: Fractional atomic coordinates and isotropic or equivalent isotropic displacement parameters $\left(\AA^{2}\right)$.

\begin{tabular}{lrrrr}
\hline Atom & $\boldsymbol{x}$ & $\boldsymbol{y}$ & $\boldsymbol{z}$ & \multicolumn{1}{c}{$\boldsymbol{U}_{\text {iso }}{ }^{*} \boldsymbol{U}_{\text {eq }}$} \\
\hline Cl1 & $0.24538(13)$ & $0.73733(6)$ & $0.50866(9)$ & $0.0438(3)$ \\
O1 & $0.2004(3)$ & $0.30936(16)$ & $0.3632(2)$ & $0.0393(5)$ \\
H1 & 0.173519 & 0.341051 & 0.286672 & $0.059^{*}$ \\
N1 & $0.1417(3)$ & $0.47566(19)$ & $0.1863(2)$ & $0.0308(5)$ \\
C1 & $0.2225(3)$ & $0.5104(2)$ & $0.4306(3)$ & $0.0270(6)$ \\
C2 & $0.2387(4)$ & $0.3907(2)$ & $0.4641(3)$ & $0.0313(6)$ \\
C3 & $0.2967(4)$ & $0.3548(3)$ & $0.6029(3)$ & $0.0373(7)$ \\
H3 & 0.307500 & 0.276026 & 0.624193 & $0.045^{*}$ \\
C4 & $0.3378(4)$ & $0.4354(3)$ & $0.7083(3)$ & $0.0401(7)$ \\
H4 & 0.378407 & 0.410409 & 0.800626 & $0.048^{*}$ \\
C5 & $0.3202(4)$ & $0.5531(3)$ & $0.6799(3)$ & $0.0380(7)$ \\
H5 & 0.345733 & 0.607048 & 0.752092 & $0.046^{*}$ \\
C6 & $0.2644(4)$ & $0.5887(2)$ & $0.5435(3)$ & $0.0309(6)$ \\
C7 & $0.1744(4)$ & $0.5486(2)$ & $0.2867(3)$ & $0.0290(6)$ \\
H7 & 0.167144 & 0.627733 & 0.267050 & $0.035^{*}$ \\
C8 & $0.1024(4)$ & $0.5202(2)$ & $0.0449(3)$ & $0.0321(6)$ \\
H8A & 0.208052 & 0.493401 & 0.005740 & $0.039^{*}$ \\
H8B & 0.105723 & 0.604428 & 0.047011 & $0.039^{*}$ \\
\hline
\end{tabular}




\section{Comment}

Schiff bases are the condensation products of primary amines and carbonyl compounds and structurally characterized by carbon-nitrogen double bond group [5-8]. Owing to the special pharmacological and physiological properties, this class of compounds has been developed as a research focus for many fields such as magnetism, catalysis, electrochemistry, chemical sensor and supramolecular chemistry etc. [9-12]. As a part of our current research interest in halogenated Schiff-base compounds, in this work, we report a new hydrazone-based compound.

The centrosymmetric title molecule is shown in the figure. There is an intramolecular hydrogen bond [O(1)$\mathrm{H}(1) \cdots \mathrm{N}(1)]$ in the asymmetric unit, which forms a $\mathrm{S}(6)$ ring plane between the phenolic oxygen atom and the diamine nitrogen atom. The $S(6)$ ring is parallel to the aryl moiety. The whole molecule exhibits a centrosymmetric "Z-type" geometry. Further analysis revealed that intermolecular $\mathrm{C}-\mathrm{H} \cdots \pi$ hydrogen bonds and aromatic face-toface $\pi-\pi$ interactions play important roles for the crystal packing. [Interactions: $\mathrm{C}(7)-\mathrm{H}(7) \cdots \mathrm{Cg}(1)$ with distance = 3.332(2) $\AA$; Cg1 $\cdots \mathrm{Cg}^{\prime}{ }^{\prime}\left({ }^{\prime}=-\mathrm{x}, 1-\mathrm{y}, 1-\mathrm{z}\right):=3.734(2)$ and Cg1...Cg1" (" = 1-x, 1-y, 1-z): 3.753(2) $\AA$ [Cg1 is the centroid of the $\mathrm{C} 1-\mathrm{C} 6$ ring]. All bond lengths and angles are in the expected ranges [13].

Author contributions: All the authors have accepted responsibility for the entire content of this submitted manuscript and approved submission.

Research funding: Fund for Yunnan Fundamental Research Projects (Grant No. 2019FH001(-091)).

Conflict of interest statement: The authors declare no conflicts of interest regarding this article.

\section{References}

1. BRUKER. SAINT, APEX2 and SADABS; Bruker AXS Inc: Madison, Wisconsin, USA, 2009.

2. Dolomanov O. V., Bourhis L. J., Gildea R. J., Howard J. A. K., Puschmann H. OLEX2: a complete structure solution, refinement and analysis program. J. Appl. Crystallogr. 2010, 42, 339-341.

3. Sheldrick G. A short history of SHELX. Acta Crystallogr. 2008, A64, 112-122.

4. Sheldrick G. Crystal structure refinement with SHELXL. Acta Crystallogr. 2015, C71, 3-8.

5. Ravikant, Kumar R. Ravikant: Review on synthesis and application of Schiff base and its transition metal complexes. Res. J. Chem. Environ. Sci. 2014, 2, 1-4.

6. Naeimi H., Safari J., Heidarnezhad A. Synthesis of Schiff base ligands derived from condensation of salicylaldehyde derivatives and synthetic diamine. Dyes Pigments 2007, 73, 251-253.

7. Liang J., Hui T., Jinrong S., Longduo Z., Qiong W. Crystal structure of rac-trans- $N, N^{\prime}$-bis(3,5-dibromosalicylidene)1,2-cyclohexanediamine, $\mathrm{C}_{20} \mathrm{H}_{18} \mathrm{Br}_{4} \mathrm{~N}_{2} \mathrm{O}_{2}$. Z. Kristallogr. NCS 2020, 235, 847-848.

8. Kumar S., Mittal S. K., Kaur N. Enhanced performance of organic nanoparticles of a Schiff base as voltammetric sensor of $\mathrm{Cu}$ (II) ions in aqueous samples. Anal. Methods 2019, 11, 359-366.

9. Hariharan P. S., Anthony S. P. Selective turn-on fluorescence for $\mathrm{Zn}^{2+}$ and $\mathrm{Zn}^{2+}+\mathrm{Cd}^{2+}$ metal ions by single Schiff base chemosensor. Anal. Chim. Acta 2014, 848, 74-79.

10. Wu Q., Pu Q., Wu Y., Shi H., He Y., Li J., Fan Q. POM-assisted synthesis of the first cyclohexanediamine-based Salen-type $\mathrm{Mn}$ III-dimer complexes. J. Coord. Chem. 2015, 68, 1010-1020.

11. Gopi D., Govindaraju K. M., Kavitha L. Investigation of triazole derived Schiff bases as corrosion inhibitors for mild steel in hydrochloric acid medium. J. Appl. Electrochem. 2010, 40, 1349-1356.

12. Klamm B. E., Windorff C. J., Marsh M. L., Meeker D. S., AlbrechtSchmitt T. E. Schiff-base coordination complexes with plutonium(IV) and cerium(IV). Chem. Commun. 2018, 54, 8634-8636.

13. Pahor N. B., Calligaris M., Nardin G., Randaccio L. N,N'-ethylenebis(salicylideneimine). Acta Crystallogr. 1978, B34, 1360-1363. 